\title{
Late renal vein aneurysm following living related renal transplant
}

\author{
Robert T. Dale, MD; Samir Bidnur, MD; Christopher Y.C. Nguan, MD, FRCSC
}

Department of Urologic Sciences, Faculty of Medicine, University of British Columbia, Vancouver, BC

Cite as: Can Urol Assoc J 2014;8(3-4):e253-5. http://dx.doi.org/10.5489/cuaj.1769 Published online April 14, 2014.

\section{Abstract}

Renal vein aneurysms are rare; there are less than 10 reported cases. As of yet there have been no reported cases of renal vein aneurysm following renal transplantation. We present a case of an incidentally discovered renal vein aneurysm following uncomplicated living related renal transplant. The lesion was discovered 4 years after the transplant through abdominal ultrasound investigation of new right lower quadrant discomfort. Magnetic resonance imaging confirmed the presence of a $2.3-\mathrm{cm}$ thrombosed renal vein aneurysm of the main renal vein. This case report highlights the rare nature of these events, the diagnostic challenges and the lack of satisfactory management guidelines in these cases.

\section{Case report}

A 57-year-old man presented with right lower quadrant discomfort 4 years following living related renal transplantation to the right iliac fossa for end-stage renal disease secondary to medication toxicity following previous bone marrow transplant. Diagnostic ultrasound failed to demonstrate any intra abdominal pathology, and the transplant kidney was normally perfused with normal resistive indices and a nominal estimated renal volume of $145 \mathrm{cc}$. There were no signs of hydronephrosis nor perinephric fluid collection and the renal artery and vein were widely patent. However, a $1.8 \times 1.8-\mathrm{cm}$ transplant renal vein aneurysm (RVA) was discovered between the hilum and primary renal vein anastomosis with the external iliac vein. Urologic consultation was sought and as the patient's clinical symptoms improved, surveillance was recommended.

A follow-up ultrasound was performed 3 months later, which again demonstrated a saccular aneurysm of the transplant renal vein measuring $2.3 \mathrm{~cm}$ with no detectable flow within it (Fig. 1). The main renal vein and artery remained patent and unremarkable with no hemodynamically significant stenosis. Subsequent magnetic resonance angiography confirmed the presence of a thrombosed venous aneurysm in the transplant renal vein (Fig. 2).

The patient was conferenced during the vascular surgery rounds where a recommendation for continued observation without systemic anticoagulation was made. Thrombosis clinic through the hematology program provided a counteropinion in favour of systemic anticoagulation to minimize risk of thrombus extension and pulmonary embolism. Given the rarity of this type of lesion, the optimal treatment course was unclear. The case was discussed with the patient who elected to proceed with anticoagulation understanding the potential risks and benefits of this strategy. The patient has been initiated on warfarin therapy with international normalization rate monitoring for target of 2.5 and continues to undergo surveillance ultrasound evaluations for this lesion.

\section{Discussion}

A review of the literature suggests there is no current consensus on treatment for RVAs, and true RVAs are rare with less than 10 total cases reported. To the best of our knowledge, there have been no reports of venous aneurysm in a transplant kidney. RVAs have been reported in the context of renal neoplastic masses, ${ }^{1}$ upper abdominal pain, ${ }^{2,3}$ or most commonly have been discovered incidentally. ${ }^{4-6}$ Sfyroeras and colleagues ${ }^{6}$ reported 198 cases of visceral venous aneurysm, of which only 6 involved the renal vein. Of the 198 cases of visceral venous aneurysms, none involved transplanted renal vessels. Abdominal venous aneurysms in general carry risks for developing complications, such as pulmonary embolism, abdominal pain, and intra-abdominal bleeding. ${ }^{5}$ Thus, in low-risk patients surgery may be indicated for large and unstable lesions.

The etiology of RVAs is unclear. A congenital defect in the venous wall architecture may predispose to aneurysm forma- 


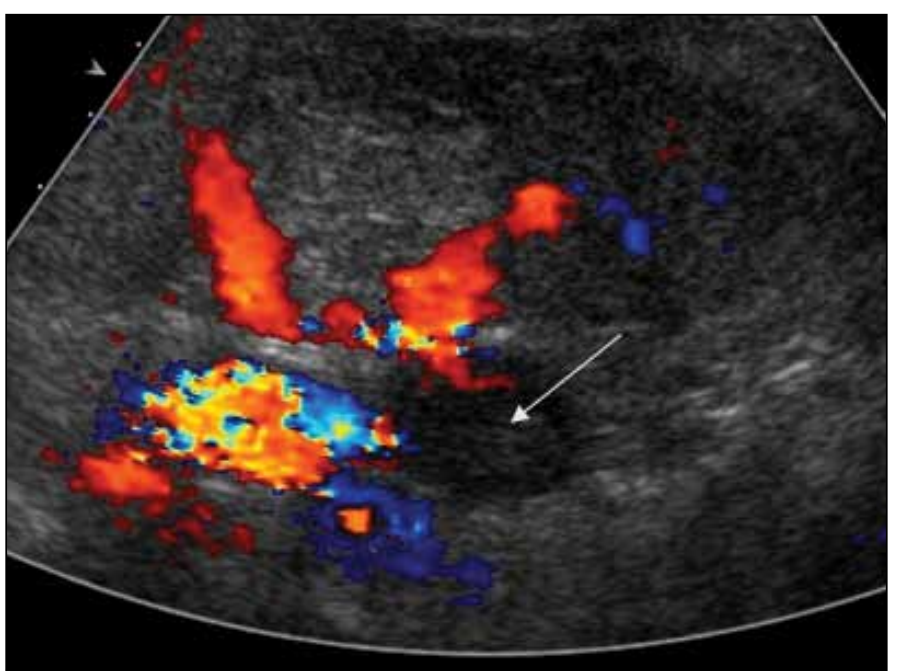

Fig. 1. Doppler ultrasound demonstrating transplant main renal vein aneurysm with no flow. The aneurysm (arrow) is visualized adjacent to the main renal vein which demonstrates normal flow patterns and velocities.

tion or an aneurysm may form secondary to hemodynamic changes in the renal vein. The latter has been described as in the "nutcracker syndrome," where compression of the left renal vein between the superior mesenteric artery and the anterior aorta can lead to venous hypertension and gonadal varices. ${ }^{7}$ Most renal vein aneurysms involve the left renal vein, which undergoes more complicated embryological development because of its increased length. ${ }^{5}$ The donor in this case underwent a left nephrectomy, although no signs of the nutcracker syndrome were present on preoperative imaging.

Diagnosis of RVAs is typically made incidentally during surgery or imaging. Barely detectable, stagnant blood flow within a venous distension is indicative of aneurysm, whereas complete absence of flow may suggest a cyst, pseudocyst, or neoplasm. ${ }^{2}$ Assessment by either Doppler sonography or renal venography can identify slow-moving blood flow in an aneurysm. ${ }^{8}$ Magnetic resonance imaging also detects slow-moving blood within a venous aneurysm. Gadolinium contrast material mixes poorly with stagnant aneurysm blood, producing a "layered gadolinium sign." However, the layered gadolinium sign was not present in this case and it may not always be identifiable in all venous aneurysms.

There is no current consensus on the optimal treatment of RVAs, and delayed venous aneurysms in a transplant allograft present unique therapeutic obstacles. Re-entering the iliac fossa following renal transplantation is technically challenging, particularly when attempting to identify and preserve the vascular hilum. Often because of the orientation of the transplant, the hilum is facing deep into the pelvis or posterior body wall, leading to a difficult approach with limited visualization. Significant injury to the renal artery, vein or ureter may result in an irreparable situation followed

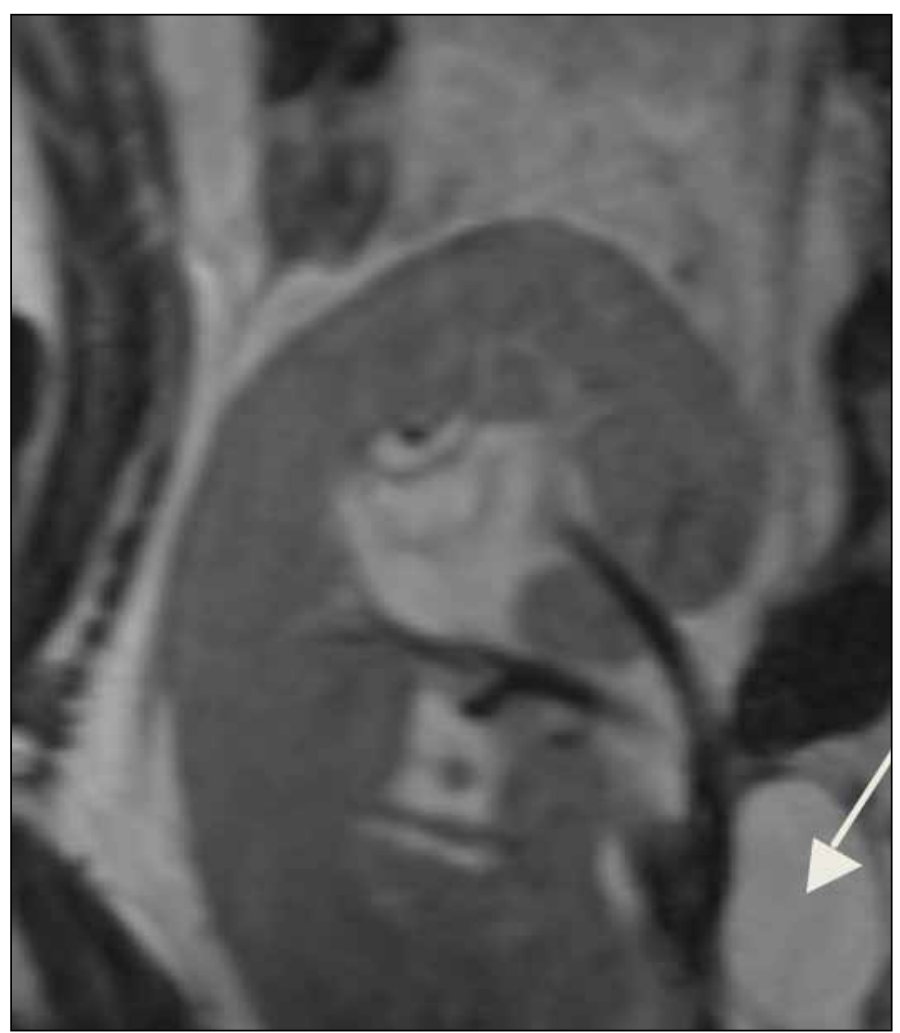

Fig. 2. T1 weighted magnetic resonance image demonstrating a patent main renal transplant vein with a saccular aneurysm. The aneurysm (arrow) measures $2.3 \mathrm{~cm}$ and is located $1.3 \mathrm{~cm}$ from the anastomosis of the main renal vein to the recipient right external iliac vein. The aneurysm demonstrates no flow and no extension of thrombus into the vein.

by compromised graft function or in the worst-case scenario, allograft nephrectomy. Likewise, injury to the recipient vascular structures, such as the iliac artery or vein, may potentially occur during dissection, again potentially endangering graft function or recipient distal extremity. Alternatively, systemic anticoagulation carries with it risks of bleeding and the need for long-term medication and monitoring, and also relegates the patient to lifelong surveillance of the graft aneurysm as it does not address the underlying problem. Radiological interventions, such as vascular coiling, have been documented for venous aneurysms in other vascular locations; however, it appears that this type of intervention is rare in renal transplantation, especially where one might consider limitation of contrast administration in a patient with limited renal reserve.

The natural history of RVA is not well understood, and threshold size, location and architectures prompting medical or surgical intervention are consequently also poorly understood. Injury to the renal vein during organ procurement, storage and handling, backtable preparation or implantation are possible times when the transplant vessels may be susceptible to injury, and hemodynamic forces following nonorthotopic anastomosis may potentially exacerbate venous injury to promote aneurysm formation. Abdominal vascular 
imaging is not a usual practice in deceased donor organ procurement processes; therefore, underlying vascular pathology may not be appreciated until after organ transplantation.

\section{Conclusion}

We describe a 57-year-old man with a $2.3-\mathrm{cm}$ saccular dilatation of the renal vein discovered by abdominal ultrasound 4 years following uncomplicated right iliac fossa living related renal transplant. A multidisciplinary approach, including urological, vascular and hematological expertise, taking into account patient wishes, can start to address the therapeutic dilemma presented by these rare lesions.

Competing interests: Dr. Dale, Dr. Bidnur and Dr. Nguan all declare no competing financial or personal interests.

This paper has been peer-reviewed.

\section{References}

1. Ferrante A, Di Stasi C, Pierconti F, et al. Incidental finding of right renal venous aneurysm in a patient with symptomatic ipsilateral renal carcinoma: A case report. Cardiovasc Pathol 2005; 14: 327-30. http:// dx.doi.org/10.1016/i.carpath.2005.06.003

2. Kabaalioglu A, Yilmaz S, Apaydin A, et al. Renal vein aneurysm: Diagnosis with color Doppler sonography. Am J Roentgenol 1997;168:645-6. http://dx.doi.org/10.2214/ajr.168.3.9057507

3. Khader SM, Saleeb SF, Teplick SK. General case of the day. Radiographics 1999;19:1683-5. http:// dx.doi.org/10.1148/radiographics.19.6.g99no121683

4. Rao MV, Polcari AJ, Sundaram V, et al. Right renal vein aneurysm discovered incidentally during laparoscopic nephrectomy. Urology 2011;77:332-3. http://dx.doi.org/10.1016/j.urology.2010.02.015

5. Lin TC, Lin CM, Chang HC, et al. A left circum-aortic renal vein aneurysm. Am I Surg 2010;200:e37-8. http://dx.doi.org/10.1016/i.amisurg.2009.05.043

6. Sfyroeras GS, Antoniou GA, Drakou AA, et al. Visceral venous aneurysms: Clinical presentation, natural history, and their managements: a systematic review. Eur I Vasc Endovasc Surg 2009;38:498-505. http://dx.doi.org/10.1016/i.ejus.2009.05.016

7. Fu W, Hong B, Gao J, et al. Nutcracker phenomenon: A new diagnostic method of multislice computed tomography angiography. Int J Urol 2006;13:870-3. http://dx.doi.org/10.1111/i.14422042.2006.01430.x

8. Irace L, Gossetti B, Benedetti-Valentini F, et al. Aneurysm of the left renal vein: A case report. J Endovasc Surg 1994;19:943-4.

9. Krinsky G, Johnson G, Rofsky N, et al. Venous aneurysms: MR diagnosis with the "layered gadolinium sign" sign. J Comput Assist Tomogr 1997;21:623-7. http://dx.doi.org/10.1097/00004728-19970700000019

Correspondence: Dr. Christopher Y.C. Nguan, 6th floor, 2775 Laurel St., Vancouver, BC V5Z 1M9; fax: 604875 5604; chris.nguan@ubcurology.com 